Filippo Sanfilippo

Carlos Corredor

Nick Fletcher

Giora Landesberg

Umberto Benedetto

Pierre Foex

Maurizio Cecconi

\section{Erratum to: Diastolic dysfunction and mortality in septic patients: a systematic review and meta-analysis}

Published online: 8 April 2015

(C) Springer-Verlag Berlin Heidelberg and ESICM 2015

The online version of the original article can be found under doi: 10.1007/s00134-015-3748-7.

F. Sanfilippo $(\varangle) \cdot$ C. Corredor · N. Fletcher

Cardiothoracic Intensive Care Unit, Intensive Care

Directorate-St Georges Healthcare NHS Trust,

London SW17 0QT, UK

e-mail: filipposanfi@yahoo.it

\section{G. Landesberg}

Department of Anesthesiology and Critical Care Medicine,

Hadassah-Hebrew University Medical Center, Jerusalem, Israel

U. Benedetto

Nuffield Department of Cardiac Surgery, John Radcliffe Hospital, Oxford OX3 9DU, UK

\section{P. Foex}

Nuffield Department of Anaesthetics, University of Oxford-John Radcliffe Hospital, Oxford OX3 9DU, UK

\section{Cecconi}

Anaesthesia and Critical Care St George's Hospital and Medical School, London SW17 0QT, UK

\section{Erratum to: Intensive Care Med}

\section{DOI 10.1007/s00134-015-3748-7}

Incorrect versions of Fig. 2a, b were supplied for publication. The authors regret any inconvenience caused and are pleased to provide the correct figures here. 
a

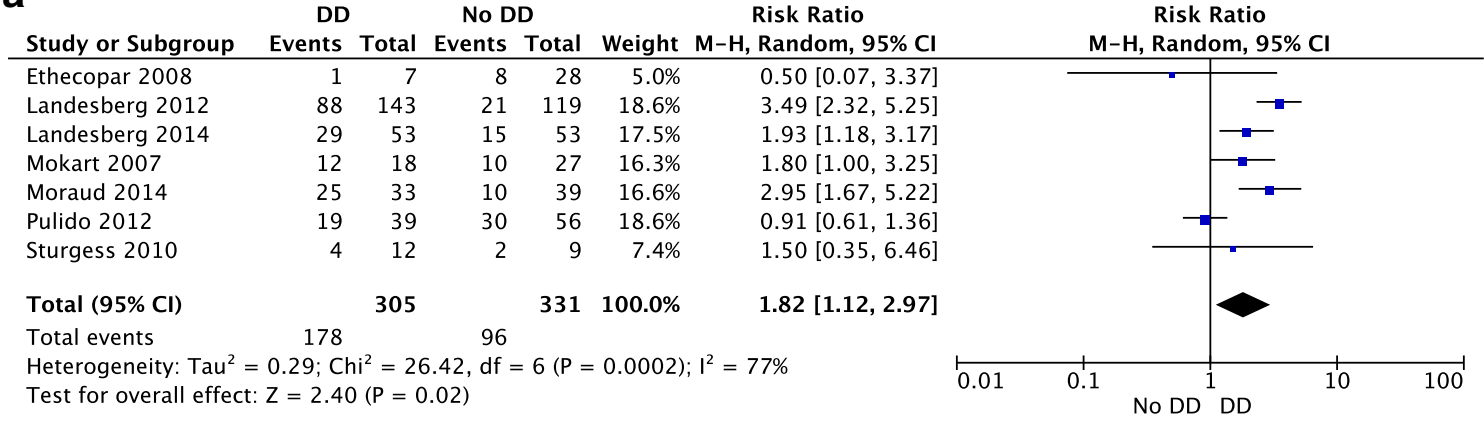

\section{b}

SD No SD Risk Ratio

Study or Subgroup Events Total Events Total Weight M-H, Random, 95\% CI

Ethecopar 2008

Landesberg 2012

Landesberg 2014

$\begin{array}{rrrrr}3 & 16 & 6 & 19 & 8.2 \% \\ 32 & 61 & 77 & 201 & 27.7 \%\end{array}$

$0.59[0.18,2.00]$

Moraud 2014

$\begin{array}{lllrr}14 & 27 & 30 & 79 & 22.9 \%\end{array}$

Pulido 2012

$\begin{array}{lllll}10 & 39 & 16 & 33 & 17.9 \%\end{array}$

$1.37[1.02,1.84]$

$1.37[0.86,2.16]$

$0.53[0.28,1.00]$

$0.73[0.47,1.14]$

Total $(95 \% \mathrm{Cl})$

Total events

172

Heterogeneity: $\mathrm{Tau}^{2}=0.13 ; \mathrm{Chi}^{2}=12.31, \mathrm{df}=4(\mathrm{P}=0.02) ; \mathrm{I}^{2}=68 \%$

Test for overall effect: $Z=0.34(P=0.73)$

$0.93[0.62,1.39]$

$409100.0 \%$

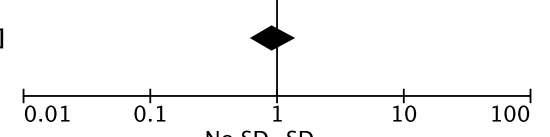

Fig. 2 a Effect of diastolic dysfunction vs no diastolic dysfunction on mortality at longest follow-up in septic patients. b Effect of systolicdysfunction vs no systolic dysfunction on mortality at longest follow-up in septic patients 